\title{
Are Humanities failing to generate Impact?
}

Dorothea Sturn, Zentrum für soziale Innovation, Vienna, sturn@zsi.at Keywords: Humanities, Impact statements, UK REF
Impact of Social Sciences and Humanities for a European Research Agenda, Pillar 3: Assessing Impact

I am particularly grateful for the assistance given by Jakob Sturn
Background

They made 1,911 submissions including:
$\mathbf{5 2 , 0 6 1}$ academic staff
$\mathbf{1 9 1 , 1 5 0}$ research outputs
$\mathbf{6 , 9 7 5}$ impact case studies

Source: REF 2014

The UK REF (Research Excellence Framework) is the system for assessing the quality of research in UK higher education institutions.

REF was initially proposed as a metrics-based evaluation to replace the Research Assessment Exercise (RAE). It was intended to reduce the administrative burden and to better present the economic and societal contribution of research (Martin 2011). However, after strong resistance from the academic community, HEFCE gave up on the switch to a bibliometrics based assessment.

A new element was introduced, however, namely narratives on impacts reached, to be drafted by the assessed institutions. Impact is defined "as an effect on, change or benefit to the economy, society, culture, public policy or services, health, the environment or quality of life, beyond academia." (HEFCE 2014, my emphasis) This means that academics are asked to show the value of their work beyond how it perpetuates the research system itself.

REF 2014 assessed nearly 7000 impact cases that cover all research areas and are grouped into the four main disciplines of Life Sciences, Sciences, Social Sciences, Arts and Humanities. The cases are published: http://impact.ref.ac.uk/CaseStudies/
Impacts and Outputs

REF 2014 recognises eight different types of impact: political, legal, health, cultural, technological, societal, economic and environmental. The two most important types are technological and societal impacts, which added together account for nearly half of all cases. Humanities show a different pattern of impact types: Cultural impacts dominate, whereas technological impacts are marginal (mostly eHumanities or computational linguistics).

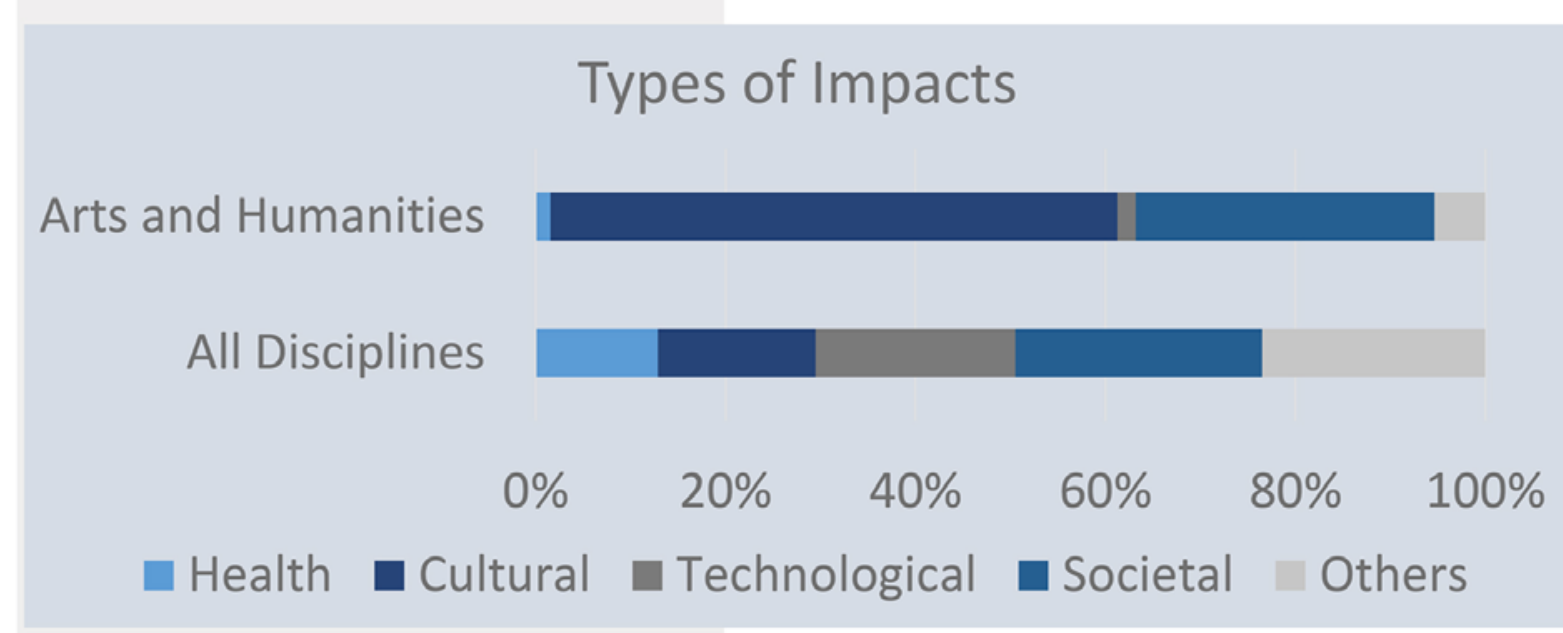

4* Impacts

\section{Characteristics of high-scored impacts}

Are there any simple recipes how to produce a $4 *$ impact? Considering the substantial financial consequences of the ratings (Reed et al 2017 calculate "something like $f 324,000$ for the most significant and far-reaching impacts"), it was only obvious that innumerable recommendations were offered on how to produce a high-score impact case (see eg Reed et al 2018). In the last two years, researchers across the UK have been mining and analysing the REF 2014 data to learn what was considered best practice. Some findings:

- Benefits should be articulated as concretely as possible (specific groups, significance and reach)

- Research (high score) and impact should be connected properly

- Impact statements should be written in a clear and plain language

Own calculation, Data Source: Impact Case Studies

\section{Successful disciplines}

The picture shows the share of best ("outstanding" $=4^{*}$ ) assessed impact and output in the four main discipline groups:

Impact generally scores higher than output (publications and other academic outputs)

- The Life Sciences score very highly on impact (esp. clinical medicine)

- Arts and Humanities show remarkably good results on outputs but fail to receive outstanding impact

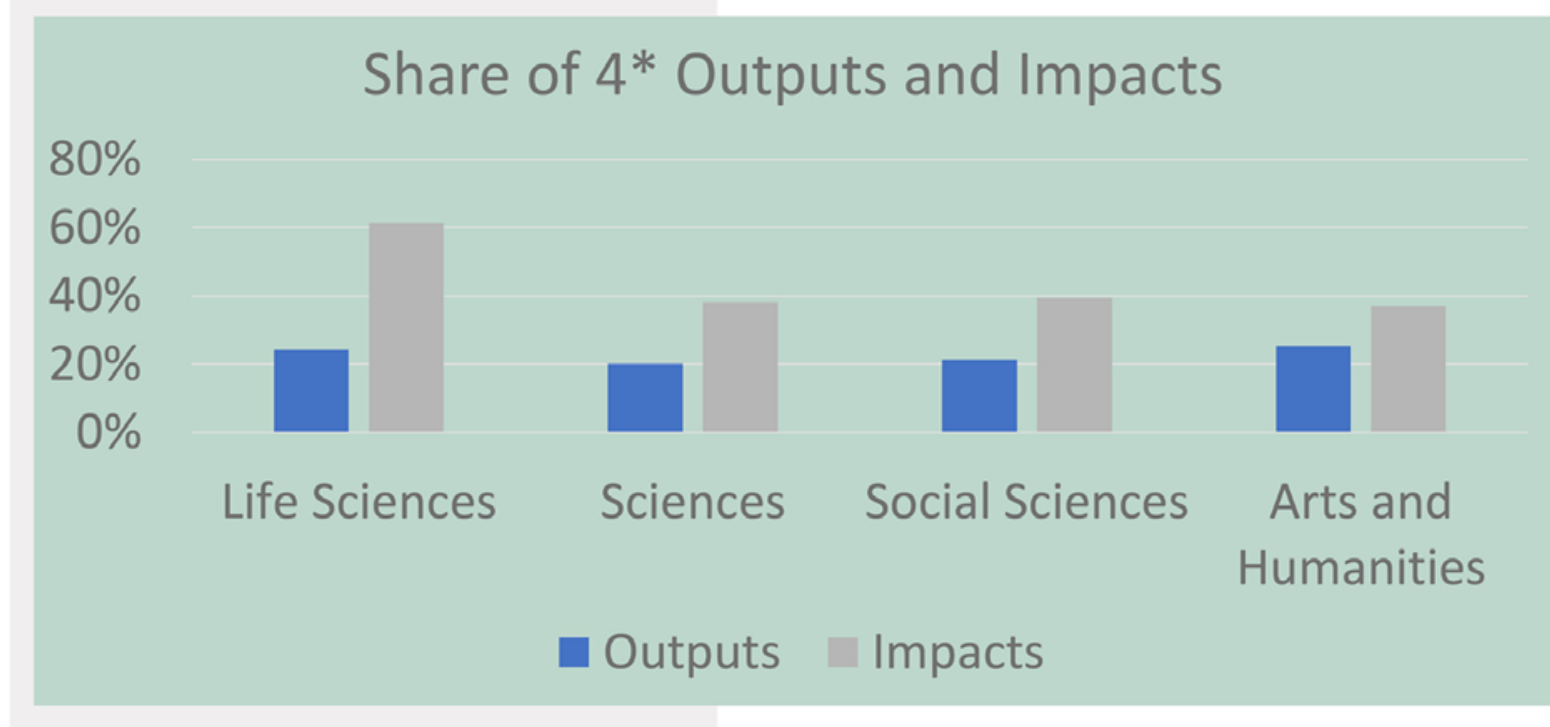

Own calculation, Data Source: Times Higher Education 2014

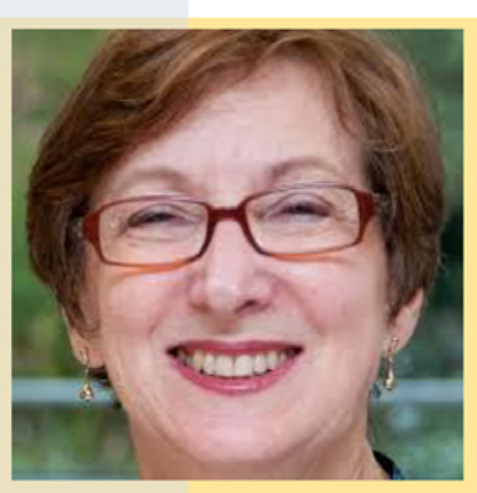

"While, traditionally, research output and impact were measured by peer-publications and citations, there is increased emphasis on a 'market-driven approach', which favours the bio-, medical and technological sciences, and has helped reinforce disciplinary hierarchy in which arts and humanities research has struggled for attention." (Hazelkorn 2015)

Using this data base as a starting point, the paper investigates the following questions:

1. Do various disciplines produce different types of impacts?

2. Are some disciplines more successful than others?

3. Is output and impact interconnected in a way that applications with highly rated outputs receive high scores for impacts as well?

4. Do high-scored impacts show characteristics different from the total amount of impacts?
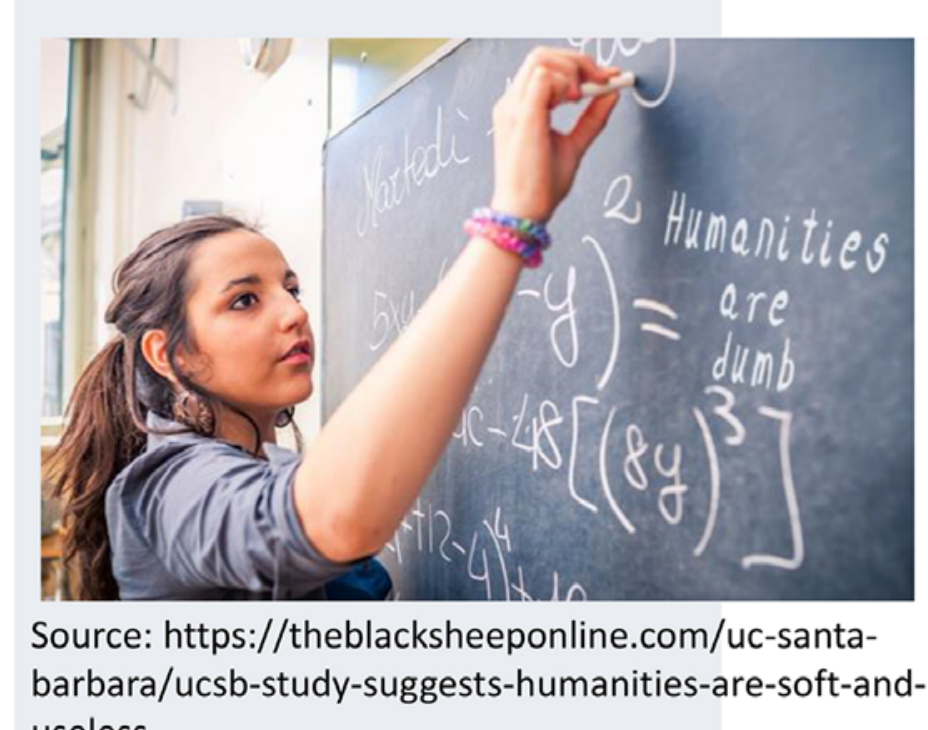

Each assessment unit's quality profile is converted into GPA by multiplying its percentage of $4^{*}$ results by 4 , its percentage of $3^{*}$ results by 3 , its percentage of $2^{*}$ added together and divided by 100 to give a score between 1 and 4 . (a)

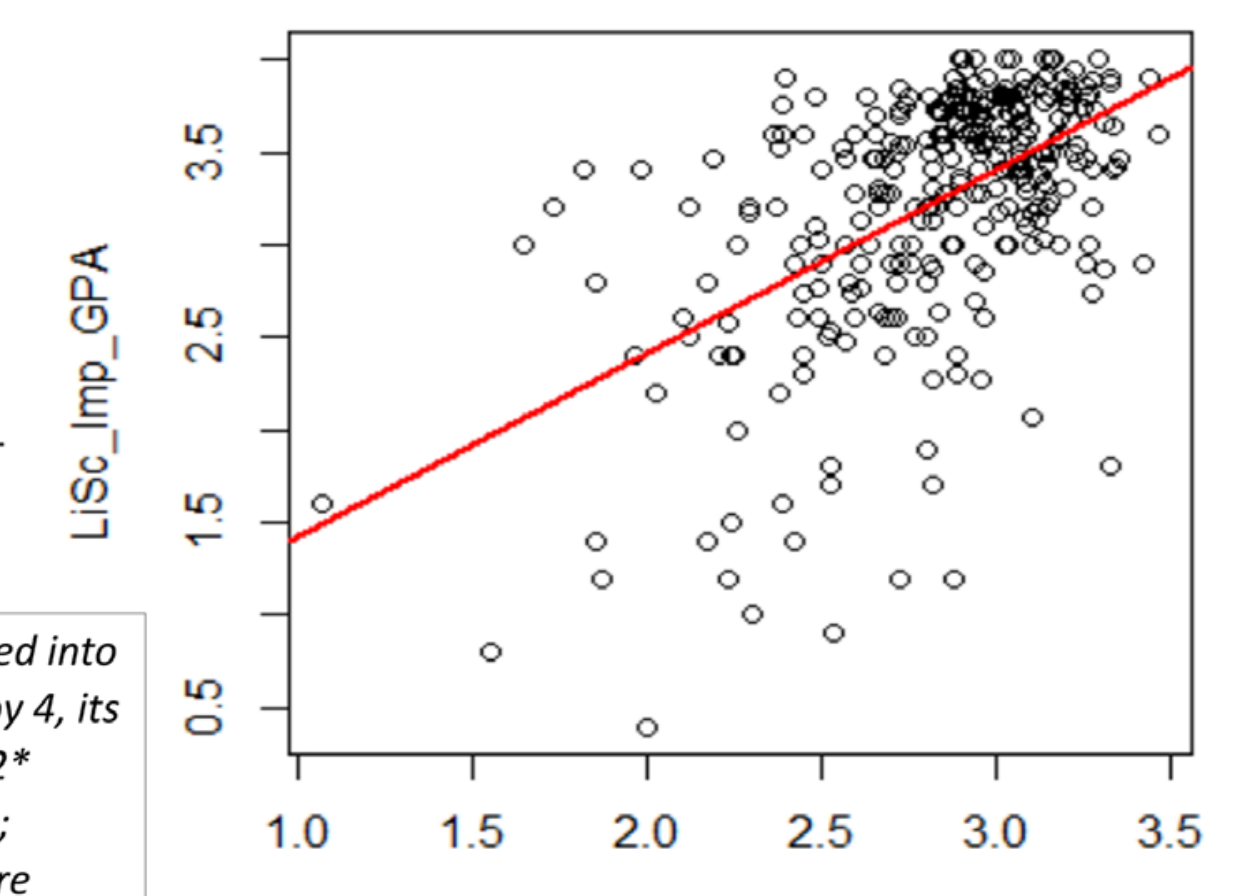

LiSc_Out_GPA
It seems that Humanities are less able to produce impact beyond academia than other disciplines, or, rather, are less able to show and communicate the impact they achieve.

A simple linear regression (Spearman's rank correlation) shows significant positive links between output and impact for all of the four disciplines. However, with $r=$ 0,36 , the connection for Humanities turns out to be much weaker than for the other three disciplines ( $r$ between 0,46 and 0,53 ).

The picture shows Life Sciences and Humanities accordin to grade point average (GPA, a weighted average of the number of stars received, see box). This means that, in general, research that scores highly on output, also receives good assessment on impact. In a recent study, Derrick et al. investigate the divergence in opinions of evaluators involved in the REF prior to the assessment. They show the wide range of views about impact as well as how research excellence (output) and impact relate to each other (Derrick et al 2016).

Own calculations, Data Source: Times Higher Education 2014

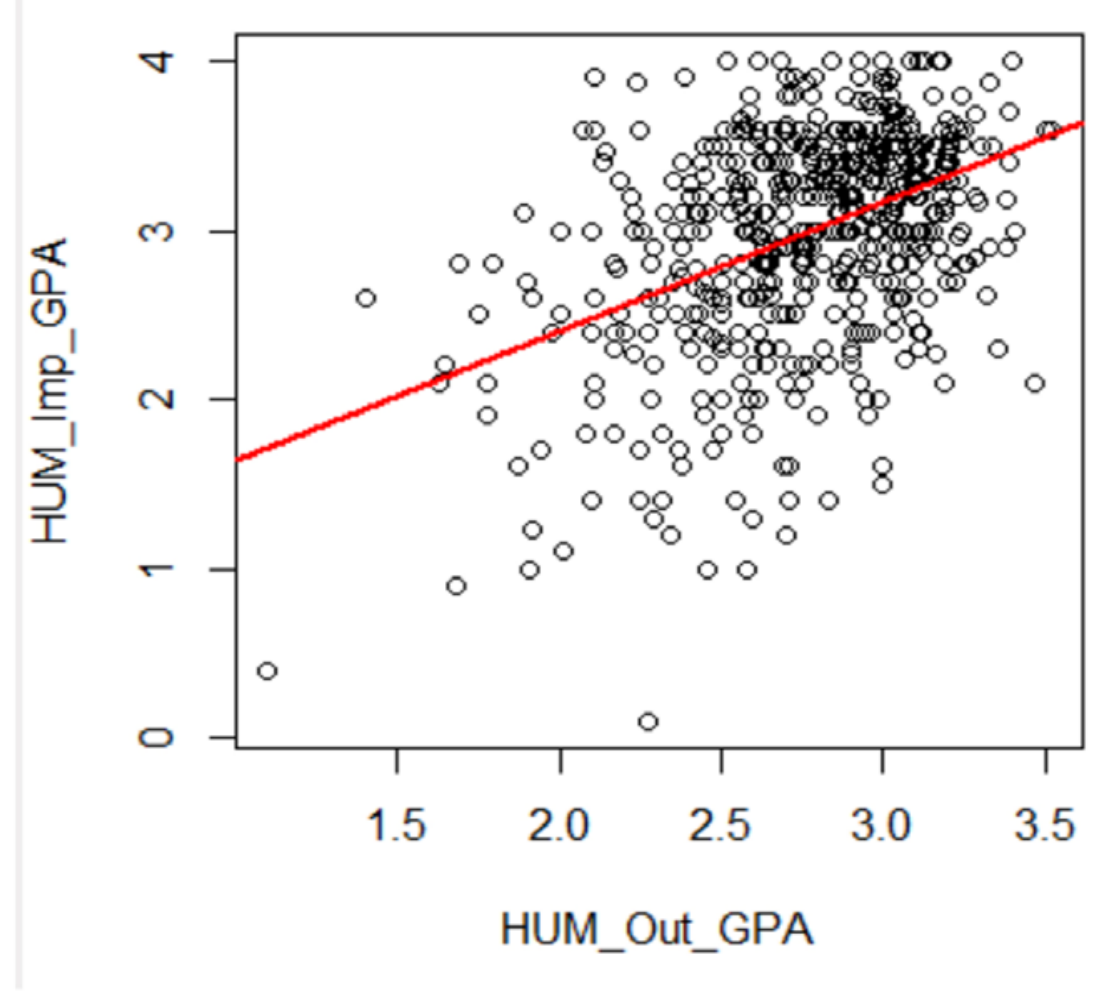

Nevertheless, my own calculation for the Arts and Humanities shows no difference between $4 *$ impact case (200 cases randomly picked) and the total amount of impact cases in the discipline group according to:

- The type of impact

- The spread of impacts over the world

- The share of interdisciplinary cases

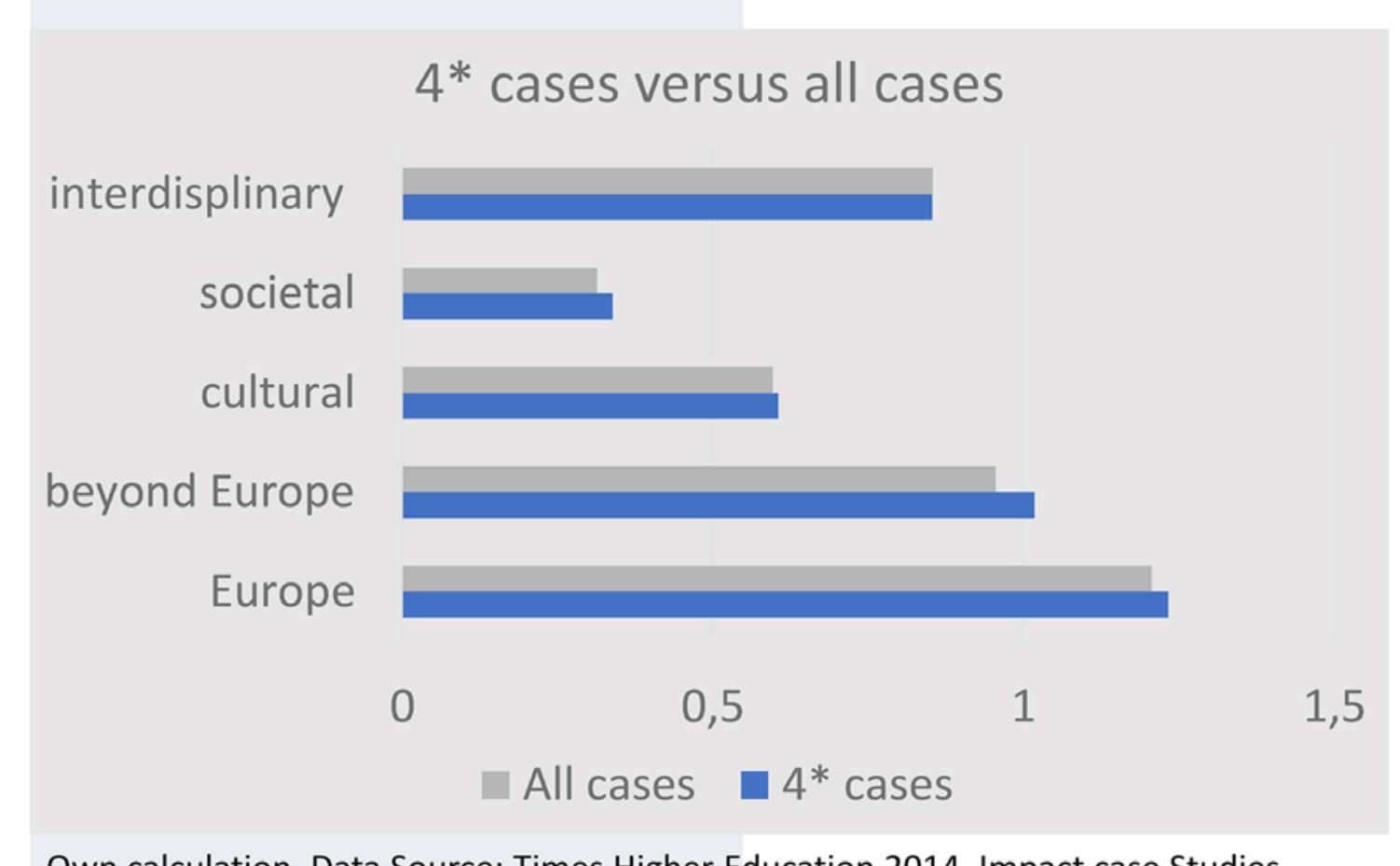

Own calculation, Data Source: Times Higher Education 2014, Impact case Studies

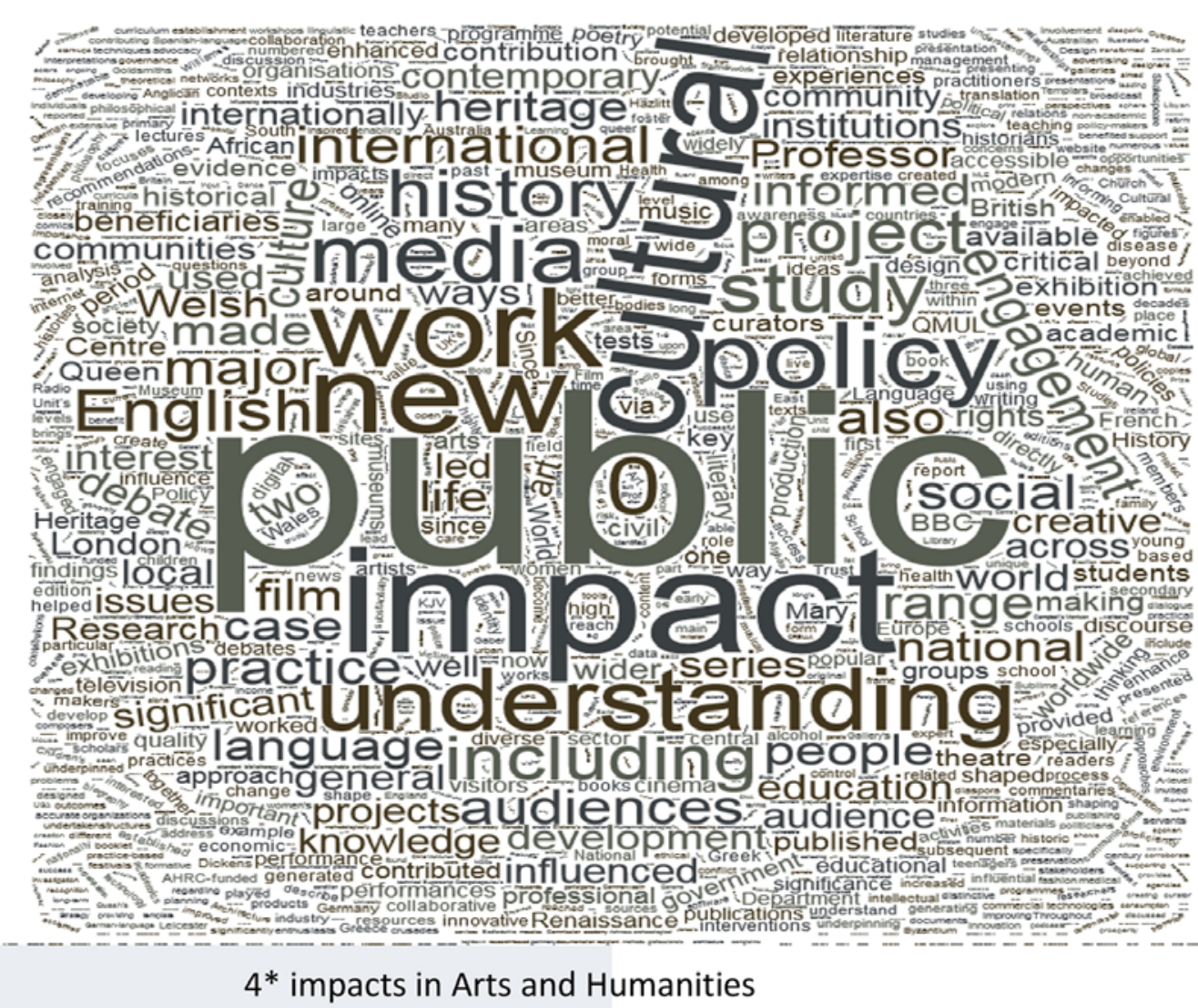

"scholars in British universities will devote less time and energy to this attempt [to deepen our understanding of "Humanities"], and more to becoming door-to-door salesmen for vulgarized versions of their increasingly market-oriented "products". (Collini 2009)

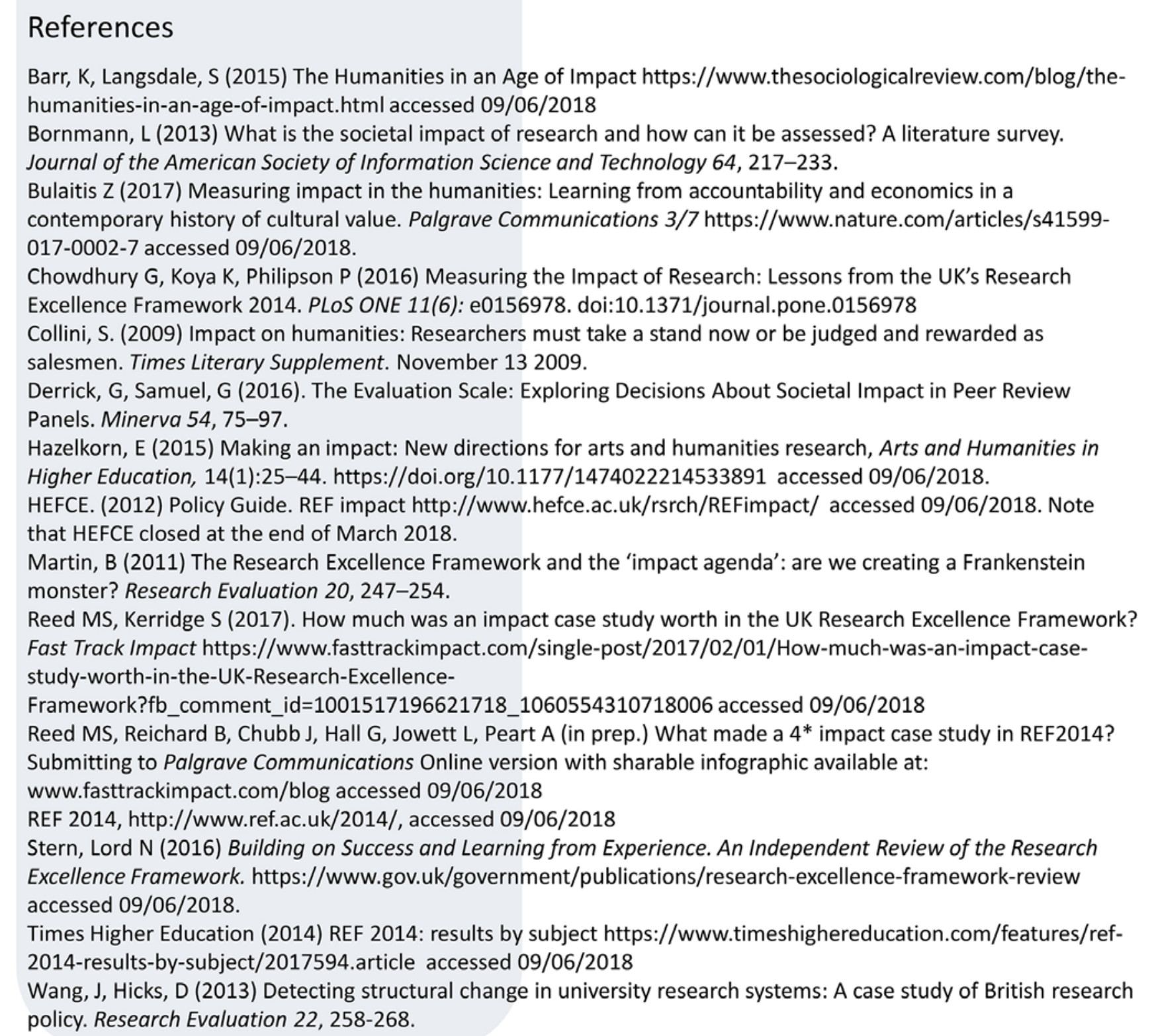

